\title{
Energy conservation and the prevalence of power distributions
}

\author{
Maciej Rybczyński a and Zbigniew Włodarczyk \\ Institute of Physics, Jan Kochanowski University, 25-406 Kielce, Poland
}

Received: 24 July 2018 / Revised: 17 September 2018

Published online: 14 November 2018

(c) The Author(s) 2018. This article is published with open access at Springerlink.com

Communicated by L. Tolos

\begin{abstract}
We analyze a connection between energy and multiplicity distributions in a statistical framework. We check the influence of energy conservation for the mechanism of particle production in relativistic proton+proton interactions. We found that energy conservation constraints may lead to the quasi-power law distributions for energy with exponent $\kappa$ depending on multiplicity distribution.
\end{abstract}

\section{Introduction}

The influence of conservation laws on particle yields has been studied for a long time. In particular, particle energy spectra within the Boltzmann-Gibbs statistics are examined and deviations from the exponential spectrum of the grand canonical ensamble are quantified and discussed [1$3]$. Let us now remember that the usual situation in statistical physics is that out of three variables considered, available energy $U$, multiplicity $N$ and temperature $T$, two are fixed and one fluctuates. Fluctuations are then given by Gamma distributions [4] (in the case of multiplicity distributions where $N$ are integers, they become Poisson distributions) and only in the thermodynamic limit, $N \rightarrow \infty$, one gets them in the form of Gaussian distributions, usually discussed in textbooks. In [4] there were situations discussed in detail when two or all three variables fluctuate. If all are fixed one has a distribution of the type

$$
f(E)=\frac{N-1}{U}\left(1-\frac{E}{U}\right)^{N-2}
$$

Equation (1) coincides with the Tsallis distribution which is based on nonextensive statistical mechanics [5, $6]$,

$$
f(E)=C\left(1+\frac{E}{\kappa \cdot T}\right)^{-\kappa},
$$

where $q=1+1 / \kappa$ is known as the nonextensivity parameter. It interpolates smoothly between the pure power-like behaviour (for $E \rightarrow 0$ ) and the exponential formulation (for $\kappa \rightarrow \infty$ ) and was first proposed in refs. [7] and [8] rediscovered in ref. [9], and widely known as QCD-based Hagedorn formula. Equation (1) has become one of the

\footnotetext{
a e-mail: maciej.rybczynski@ujk.edu.pl
}

standard phenomenological formulas used in data analysis of multiparticle production processed, cf. review [10] and references therein.

Quasi power-law distributions are ubiquitous in many branches of science and their possible origins are accordingly numerous. Among many possibilities (cf. [10-12] for more details and references) the most interesting is the socalled superstatistics, stochastic network, and connection with multiplicative noise. In this paper we discuss the influence of conservation laws on multiparticle production processes, forcing the use of conditional probabilities and resulting in quasi power-law distributions.

\section{An influence of energy conservation on the particle production mechanism}

The proper implementation of energy conservation in Monte Carlo simulations is still an open question. In the following we review the possible attempts, deriving the energy distributions when the ensemble observes an energy conservation constraint.

\subsection{Conditional probability}

Let $\left\{E_{1, \ldots, N}\right\}$ be a set of $N$ independent identically distributed random variables described by some parameter (temperature $T$ ). For independent energies $E=E_{i=1, \ldots, N}$ each distributed according to the Boltzmann distribution,

$$
f_{1}(E)=\frac{1}{T} \exp \left(-\frac{E}{T}\right),
$$


the sum $W=\sum_{i=1}^{N} E_{i}$ is then distributed according to the gamma distribution,

$$
f_{N}(W)=\frac{1}{T \cdot \Gamma(N)}\left(\frac{W}{T}\right)^{N-1} \exp \left(-\frac{W}{T}\right)
$$

If the available energy $U=$ const is limited then we have the following conditional probability for the single-particle distribution:

$$
f(E \mid U)=\frac{f_{1}(E) \cdot f_{N-1}(U-E)}{f_{N}(U)}=\frac{N-1}{U}\left(1-\frac{E}{U}\right)^{N-2} .
$$

In the above consideration the value of the $T$ parameter is given $b^{1}$

$$
T=\langle E\rangle=\frac{U}{N}
$$

and one can rewrite eq. (5) in the form

$$
f(E \mid U)=\frac{N-1}{N \cdot T}\left(1-\frac{E}{N \cdot T}\right)^{N-2}
$$

which only in the limit $N \rightarrow \infty$ tends to the Boltzmann distribution, $\lim _{N \rightarrow \infty} f(E \mid U)=f_{1}(E)$.

\subsection{Rescaling approach}

Sampling $\left\{E_{1, \ldots, N}^{\prime}\right\}$ from exponential distribution, their sum $W=\sum_{i=1}^{N} E_{i}^{\prime}$ and energy conservation imposes the constraint $U=\sum_{i=1}^{N} E_{i}$ The rescaling technique outlined in ref. [13] shows that, by imposing energy conservation, we actually expect the following sequence of re-scaled energies:

$$
E_{i}=E_{i}^{\prime} \frac{U}{W}
$$

The strategy followed here is based on the partition through renormalization and works basically as follows: out of an interval $[0,1]$, a partition is performed by choosing $N$ random numbers between 0 and 1 and then renormalizing them such that their sum is equal to unity [14]. Such strategy can be seen as a particular choice of Dirichlet partition of the interval [15].

If $X_{i}$ are independently distributed exponential random variables then for $k<N$ a ratio

$$
r=\frac{\sum_{i=1}^{k} X_{i}}{\sum_{i=1}^{N} X_{i}}
$$

has the same distribution as the $k$-th smallest number of $(N-1)$ numbers chosen independently and uniformly

\footnotetext{
${ }^{1}$ In the relativistic regime, where masses are negligible in comparison with momenta, $p \ll m$, and for the cylindrical phase space in which $p_{L} \gg p_{T}$ we have $E \sim p_{L}$. This simplification (one-dimensional space) leads to a simple interpretation of temperature parameter, $T=\langle E\rangle$.
}

between 0 and 1 . This is also known as the Beta distribution $[15]$

$$
f_{k}(r)=\frac{\Gamma(N)}{\Gamma(k) \Gamma(N-k)} r^{k-1}(1-r)^{N-k-1} .
$$

For $k=1$ and $E=r \cdot U$ we have eq. (5).

\subsection{Induced partition}

Actually, such distribution emerges directly from the calculus of probability for a situation known as induced partition [16]. In short: $N-1$ randomly chosen independent points $\left\{U_{1}, \ldots, U_{N-1}\right\}$ split a segment $(0, U)$ into $N$ parts, whose length is distributed according to eq. (5). The length of the $k$-th part corresponds to the value of energy $E_{k}=U_{k+1}-U_{k}$ (for ordered $U_{k}$ ). In our case it could correspond to the case of random breaks of a string in $N-1$ points in the energy space ${ }^{2}$.

In this work we use an induced partition scenario to ensure energy conservation in Monte Carlo simulations.

\section{Energy conservation in ensemble with fluctuating multiplicity}

Whereas for fixed $N$ one has

$$
f(E, N)=\frac{N-1}{U}\left(1-\frac{E}{U}\right)^{N-2}
$$

then for $N$ fluctuating according to $P(N)$, the resulting distribution is

$$
f(E)=\sum_{N} f(E, N) \cdot P(N)
$$

For $P(N)$ given by the binomial distribution, Poisson distribution and negative binomial distribution, eq. (12) leads to the Tsallis distribution given by eq. (2) [19].

Note that for all cases

$$
\frac{1}{\kappa} \simeq \frac{\operatorname{Var}(N)}{\langle N\rangle^{2}}-\frac{1}{\langle N\rangle},
$$

with equality when the replacing $N-2$ by $N$ in eq. (11). It is natural that for the Poisson distribution $\left(\kappa^{-1}=0\right)$ the energy distribution given by eq. (2) reproduces the Boltzmann-Gibbs statistics.

\section{Results}

In order to test numerically the above considerations we checked the influence of energy conservation for the mechanism of particle production in relativistic proton+proton

\footnotetext{
2 Note that the induced partition differs from a successive sampling from uniform distribution, $E_{k} \in\left[0, U-\sum_{i=1}^{k-1} E_{i}\right]$ which results in $f(E) \sim 1 / E[17,18]$.
} 
Table 1. Parameters of multiplicity distributions used for simulation.

\begin{tabular}{cccccc}
\hline$\sqrt{s}[\mathrm{GeV}]$ & $\left\langle N_{\text {neg }}\right\rangle[20]$ & $k_{\text {neg }}[20]$ & $\operatorname{Var}\left(N_{\text {neg }}\right)$ & $\langle N\rangle$ & $\operatorname{Var}(N)$ \\
\hline 23.9 & 3.62 & 33.0 & 4.02 & 10.87 & 20.12 \\
27.6 & 3.92 & 12.8 & 5.12 & 11.76 & 25.6 \\
30.4 & 4.27 & 13.3 & 5.64 & 12.8 & 28.18 \\
44.0 & 5.04 & 11.4 & 7.27 & 15.12 & 36.33 \\
52.6 & 5.4 & 9.4 & 8.47 & 16.15 & 42.33 \\
62.2 & 5.8 & 9.2 & 9.5 & 17.45 & 47.47 \\
200 & 9.7 & 4.7 & 29.7 & 29.1 & 148.6 \\
540 & 13.2 & 3.6 & 61.0 & 39.4 & 305.2 \\
900 & 16.3 & 3.1 & 102.0 & 48.9 & 510.0 \\
\hline
\end{tabular}

interactions. For the simulations we used parameters stored in table 1. Data on multiplicity distributions of particles produced in proton+proton non-diffractive events at wide range of energies, $23.9<\sqrt{s}<900 \mathrm{GeV}$ (the average value of produced negatively charged particles, $\left\langle N_{n e g}\right\rangle$ and $k_{n e g}$ parameter of a negative binomial distribution fit to the measured multiplicity distributions) were taken from ref. [20].

Variances of the multiplicity distributions of negatively charged particles were calculated from the formula

$$
\operatorname{Var}\left(N_{n e g}\right)=\frac{\left\langle N_{n e g}\right\rangle^{2}}{k_{n e g}}+\left\langle N_{n e g}\right\rangle
$$

Since we simulate neutral as well as charged particles, then $\langle N\rangle=3 \cdot\left\langle N_{n e g}\right\rangle$ and $\operatorname{Var}(N)=5 \cdot \operatorname{Var}\left(N_{\text {neg }}\right)$ (see footnote $\left.^{3}\right)$.

Due to the well-known fact that, on average, a half of the center-of-mass energy, $\sqrt{s}$ is spent for a production of secondary particles [22] we share, using the induced partition mechanism described in sect. 2.3, all available energy $U=\sqrt{s} / 2$ among produced particles. We always assume the production of pions (with mass $m=0.139 \mathrm{GeV}$ ) as secondary particles, thus

$$
U=\sum_{i=1}^{N} E_{i}
$$

where $E_{i} \in(m, \sqrt{s} / 2)$.

We consider three different variants of generation of the number of particles $N$ in a given event:

- constant number of particles, $N=\operatorname{nint}(\langle N\rangle)$, where the function nint() returns the integer value closer to a real number being its argument,

\footnotetext{
${ }^{3}$ Due to electric charge conservation, in a single event we have the same number of positively and negatively charged particles, $N_{\text {pos }}=N_{\text {neg }}$. For simplicity we assume that $\left\langle N_{n e g}\right\rangle=$ $\left\langle N_{\text {pos }}\right\rangle=\left\langle N_{0}\right\rangle$, where $N_{0}$ stands for a number of electrically neutral particles produced in a single collision. We assume also $\operatorname{Var}\left(N_{0}\right)=\operatorname{Var}\left(N_{n e g}\right)$. For a detailed discussion of correlations induced by conservation laws see [21].
}

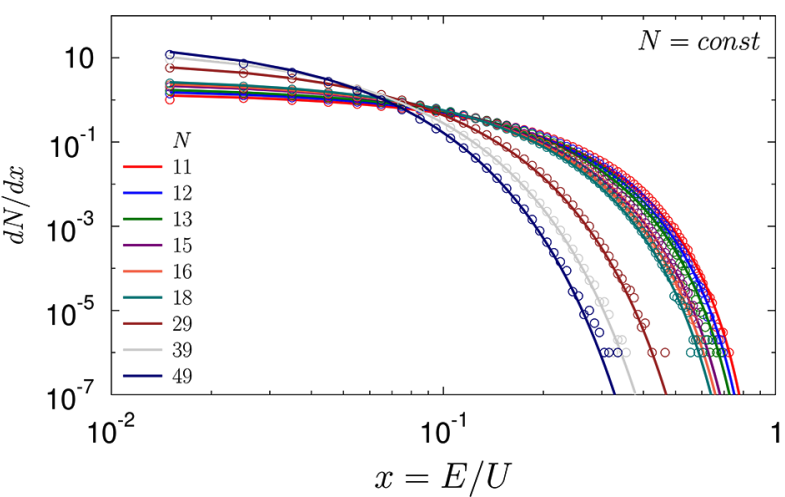

Fig. 1. (Color online) Energy distributions of particles in the case of their constant number in each simulated event. Results of simulations are fitted by eq. (16). See text for details.

- number of particles generated from Poisson distribution, $N=\operatorname{pois}(\langle N\rangle)$,

- number of particles generated from negative binomial distribution, $N=$ negbin $(\langle N\rangle, \operatorname{Var}(N))$.

In the following subsections we show the corresponding results of the simulations.

\subsection{Constant number of particles}

For each event we take a constant number of particles, $N=\operatorname{nint}(\langle N\rangle)$, where the function $\operatorname{nint}()$ returns the integer value closest to a real number being its argument. For the fit of the energy distribution of secondary particles, we use [19]

$$
f(E)=C \cdot\left(1-\frac{E}{U^{\prime}}\right)^{n-2},
$$

where $C$ is a normalization factor, $n$ is a fit parameter, and $U^{\prime}=U-N \cdot m$ is an available "kinetic" energy distributed among secondary particles.

In fig. 1 we show the obtained energy distributions of secondary particles fitted by eq. (16). We note for all considered reactions the fit parameter $n=N$.

\subsection{Number of particles generated from the Poisson distribution}

In this case for each event the number of particles is sampled from the Poisson distribution,

$$
P(N)=\frac{\langle N\rangle^{N}}{N !} \exp (-\langle N\rangle) .
$$

The obtained energy distributions of secondary particles were fitted by the following formula [19]:

$$
f(E)=C \cdot \exp \left(-\frac{n \cdot E}{U}\right),
$$

where $C$ is a normalization factor, and $n$ is a fit parameter.

Figure 2 presents the resulting energy distributions of secondary particles fitted by eq. (18). In this case, for all considered reactions the fit parameter $n=\langle N\rangle+1$. 


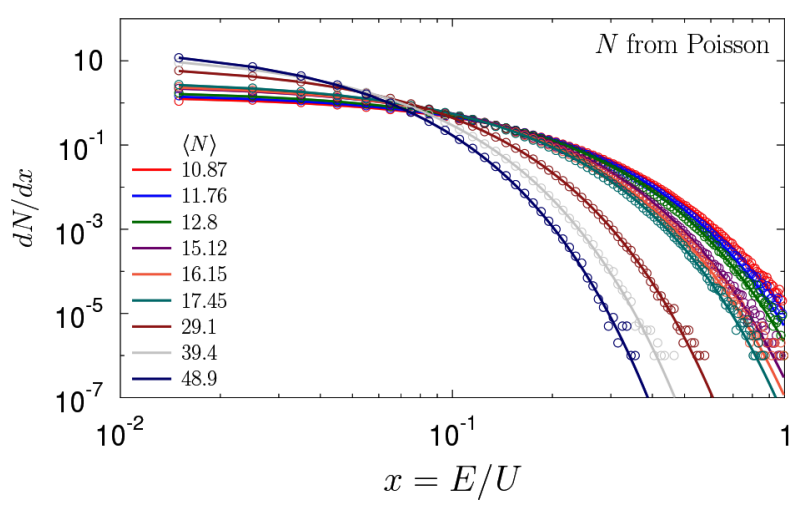

Fig. 2. (Color online) Energy distributions of particles in the case where the number of particles in each event is taken from the Poisson distribution. Results of simulations are fitted by eq. (18).

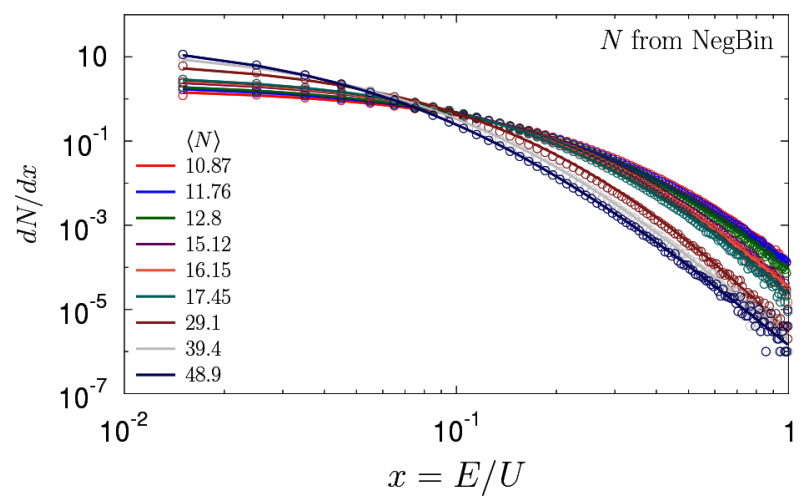

Fig. 3. (Color online) Energy distributions of particles in the case where the number of particles in each event is taken from a negative binomial distribution. Results of simulations are fitted by eq. (2).

\subsection{Number of particles generated from the negative binomial distribution}

Now let us consider a case where in each simulated event the number of particles is sampled from a negative binomial distribution,

$$
P(N)=\frac{\Gamma(N+k)}{\Gamma(N+1) \cdot \Gamma(k)}\left(\frac{\langle N\rangle}{k}\right)^{N}\left(1+\frac{\langle N\rangle}{k}\right)^{-k-N}
$$

with $\operatorname{Var}(N)=\langle N\rangle^{2} / k+\langle N\rangle$.

For the fit of the obtained energy distributions of secondary particles, we use Tsallis distribution given by eq. (2) with $C$ being a normalization factor and $\kappa$ and $T$ fit parameters.

In fig. 3 we show the corresponding energy distributions of secondary particles fitted by eq. (2). In table 2 we present the values of parameters obtained from the fit.

We report a substantial decrease of the values of our $\kappa$ parameter extracted from the fits done by eq. (2) as a function of $\sqrt{s}$, especially for the higher considered centerof-mass energies, which is presented in fig. 4 . The $\kappa$ val-
Table 2. Parameters of fits done by eq. (2) to the simulated energy distributions in the case where the number of particles is sampled from a negative binomial distribution. The values of $k$ parameters are extracted from the multiplicity distributions given by eq. (19).

\begin{tabular}{cccc}
\hline$\sqrt{s}[\mathrm{GeV}]$ & $\kappa$ & $T$ & $k$ \\
\hline 23.9 & 10.1 & 0.749 & 13.2 \\
27.6 & 9.0 & 0.78 & 10.2 \\
30.4 & 9.0 & 0.796 & 10.8 \\
44 & 10.1 & 1.034 & 10.8 \\
52.6 & 9.5 & 1.16 & 10.1 \\
62.2 & 9.7 & 1.29 & 10.2 \\
200 & 9.0 & 2.87 & 7.1 \\
540 & 8.0 & 5.23 & 5.9 \\
900 & 7.2 & 6.7 & 5.2 \\
\hline
\end{tabular}

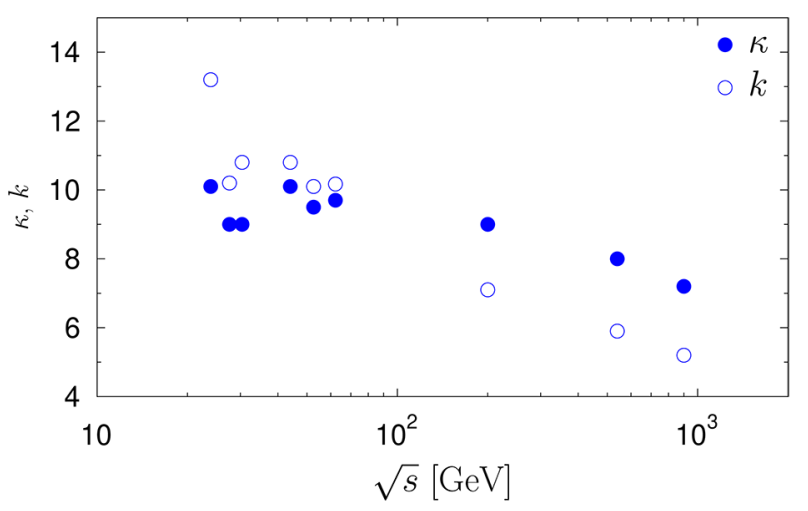

Fig. 4. (Color online) Center-of-mass energy $\sqrt{s}$ dependence of $\kappa$ and $k$ parameters.

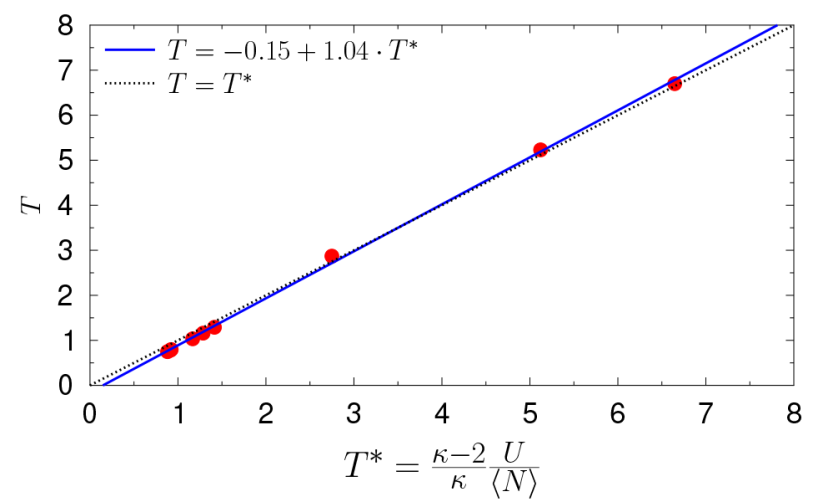

Fig. 5. (Color online) $T$ parameter as a function of the effective temperature $T^{*}$.

ues obtained from the energy distributions are lower (nonextensivity parameters $q=1+1 / \kappa$ are higher) than those evaluated from data on transverse momentum distributions [23]. The observed decrease of $\kappa$ with energy is a consequence of broadening of multiplicity distributions (decreasing of $k$ parameter with energy). Tsallis distribution is strongly connected with the negative binomial distribution. In the induced partition scenario with $N$ fluctuating 
according to the negative binomial distribution we expect (in an ideal case) $\kappa=k$. Such correspondence between energy distribution and multiplicity distribution was reported also from a superstatistical point of view [24].

Following eq. (6) we expect a linear dependence of the obtained values of $T$ parameters as a function of the socalled effective temperature $T^{*}$ given by

$$
T^{*}=\frac{\kappa-2}{\kappa} \frac{U}{\langle N\rangle},
$$

where $\kappa$ is taken from the fits to our simulated energy distributions done by eq. (2). In fact, we obtained such linear behaviour,

$$
T=1.04 \cdot T^{*}-0.15,
$$

which is presented in fig. 5 .

For the Tsallis distribution, eq. (2), the average energy is equal to

$$
\langle E\rangle=\frac{\kappa}{\kappa-2} T
$$

and we have the relation

$$
T^{*}=T \frac{U}{\langle N\rangle\langle E\rangle},
$$

which for $U=\langle N\rangle\langle E\rangle$ results in the equality $T=T^{*}$. The evaluated $T\left(T^{*}\right)$ dependence shows only a small deviation from such equality at low temperature parameters.

\section{Concluding remarks}

In this paper we approach the hadronization problem from a statistical point of view. We assume that the available phase-space volume at a given total energy, $U$, is filled in statistical equilibrium. We demonstrate that energy conservation constraints may lead to quasi-power law distributions. Our results are as follows:

- The case of a constant number of particles in each collision leads to the energy distributions described by eq. (16) with a parameter $n$ equal to the number of particles $N$ generated in the event, for each considered reaction.

- In the case of a fluctuating number of particles in each event according to the Poisson distribution, the conservation of energy leads to energy distributions of secondary particles given by eq. (18) with a parameter $n=\langle N\rangle+1$.

- When the number of particles is sampled from a negative binomial distribution, the energy conservation leads to the Tsallis-Pareto stationary distribution [5, $6]$ given by eq. (2). A connection between $E$ and $N$ distributions is hinted at in different $\kappa=1 /(q-1)$ values for different particle numbers.
In addition to the numerous possible origins of quasi power-law distributions [10-12] we show that energy conservation in natural way leads to the Tsallis distribution with exponent determined by multiplicity distribution. There is a remarkable correspondence between this scenario and superstatistics. Boltzmann distribution for energy is associated with Poisson distribution for multiplicity. Fluctuating parameter $1 / T$ according to Gamma distribution we obtain Tsallis distribution for energy and negative binomial distribution for multiplicity [24]. On the other hand, the fluctuation of $\langle N\rangle$ in the Poisson distribution (according to Gamma distribution) leads to negative binomial distribution for multiplicity and Tsallis distribution for energy.

The numerical simulations were carried out in laboratories created under the project "Development of research base of specialized laboratories of public universities in Swietokrzyskie region", POIG 02.2.00-26-023/08, 19 May 2009. MR was supported by the Polish National Science Centre (NCN) grant 2016/23/B/ST2/00692.

Open Access This is an open access article distributed under the terms of the Creative Commons Attribution License (http://creativecommons.org/licenses/by/4.0), which permits unrestricted use, distribution, and reproduction in any medium, provided the original work is properly cited.

\section{References}

1. V.V. Begun, M. Gazdzicki, M.I. Gorenstein, Phys. Rev. C 78, 024904 (2008) arXiv:0804.0075 [hep-ph].

2. V.V. Begun, M. Gazdzicki, M.I. Gorenstein, Acta Phys. Pol. B 43, 1713 (2012) arXiv:1201.5843 [nucl-th].

3. M. Rybczyński and Z. Włodarczyk, Eur. Phys. J. A 51, 80 (2015) arXiv:1505.03663 [hep-ph].

4. G. Wilk, Z. Wlodarczyk, Physica A 390, 3566 (2011) arXiv:1102.1824 [cond-mat.stat-mech].

5. C. Tsallis, J. Stat. Phys. 52, 479 (1988).

6. C. Tsallis, Introduction to Nonextensive Statistical Mechanics: Approaching a Complex World (Springer, New York, 2009).

7. C. Michael, L. Vanryckeghem, J. Phys. G 3, L151 (1977).

8. C. Michael, Prog. Part. Nucl. Phys. 2, 1 (1979).

9. R. Hagedorn, Riv. Nuovo Cimento 6, N. 10 (1983).

10. G. Wilk, Z. Włodarczyk, Int. J. Mod. Phys. A 33, 1830008 (2018) arXiv:1803.07832 [hep-ph].

11. G. Wilk, Z. Wlodarczyk, Eur. Phys. J. A 40, 299 (2009) arXiv:0810.2939 [hep-ph].

12. G. Wilk, Z. Wlodarczyk, Eur. Phys. J. A 48, 161 (2012) arXiv:1203.4452 [hep-ph].

13. B. Corominas-Murtra, R. Hane, S. Thurner, Sci. Rep. 7, 11223 (2017).

14. J.F.C. Kingman, Poisson Processes, Oxford Studies in Probability, Vol. 3 (The Clarendon Press, Oxford University Press, New York, 1993).

15. T. Huillet, Commun. Stat. Theory Methods 34, 1019 (2005). 
16. W. Feller, An Introduction to Probability Theory and Its Applications, Vol. II (John Wiley and Sons Inc., New York, 1966).

17. M. Shibata, Phys. Rev. D 24, 1847 (1981).

18. C.Y. Wong, Phys. Rev. D 30, 972 (1984).

19. G. Wilk, Z. Włodarczyk, Acta Phys. Pol. B 46, 1103 (2015) arXiv:1501.01936 [cond-mat.stat-mech].

20. R. Szwed, G. Wrochna, A.K. Wroblewski, Acta Phys. Pol. B 19, 763 (1988).
21. V.V. Begun, M. Gazdzicki, M.I. Gorenstein, Phys. Rev. C 80, 064903 (2009) arXiv:0812.3078 [hep-ph].

22. F.S. Navarra, O.V. Utyuzh, G. Wilk, Z. Wlodarczyk, Phys. Rev. D 67, 114002 (2003) arXiv:hep-ph/0301258.

23. T. Wibig, J. Phys. G 37, 115009 (2010) arXiv:1005.5652 [hep-ph].

24. G. Wilk, Z. Wlodarczyk, Physica A 376, 279 (2007) arXiv:cond-mat/0603157. 